\title{
Phage Particles as Vaccine Delivery Vehicles: Concepts, Applications and Prospects
}

\author{
Narjes Jafari ${ }^{1}$, Saeid Abediankenari ${ }^{2 *}$
}

\begin{abstract}
The development of new strategies for vaccine delivery for generating protective and long-lasting immune responses has become an expanding field of research. In the last years, it has been recognized that bacteriophages have several potential applications in the biotechnology and medical fields because of their intrinsic advantages, such as ease of manipulation and large-scale production. Over the past two decades, bacteriophages have gained special attention as vehicles for protein/peptide or DNA vaccine delivery. In fact, whole phage particles are used as vaccine delivery vehicles to achieve the aim of enhanced immunization. In this strategy, the carried vaccine is protected from environmental damage by phage particles. In this review, phage-based vaccine categories and their development are presented in detail, with discussion of the potential of phage-based vaccines for protection against microbial diseases and cancer treatment. Also reviewed are some recent advances in the field of phagebased vaccines.
\end{abstract}

Keywords: Bacteriophage - vaccine - carrier - immune response

Asian Pac J Cancer Prev, 16 (18), 8019-8029

\section{Introduction}

Nowadays, vaccines are defined as biological agents, which are prepared by disease-causing bacteria, viruses, and so on, to stimulate the host's immune system for protection against a disease and for treatment. Conventional vaccines, which are based on live attenuated or inactivated microorganisms, have made great progress, and some of them have been used in clinical application (Yang et al., 2015). However, many pathogenic microorganisms are difficult to culture in vitro, and therefore production of live attenuated or inactivated vaccines of these pathogens is impossible (Purcell et al., 2007), these vaccines could also pose potential risks because the pathogen may contain material that initiates unwanted host responses (Purcell et al., 2007; Yang et al., 2015). Moreover, in vaccine design for cancer using whole cell, although a limited set of antigens is suitable to cancer vaccination, tumor cells or lysed may contain predominantly normal self-proteins that are of no therapeutic benefit, or contain material that potentially caries the capacity to induce further malignancy (Purcell et al., 2007). Therefore, the identification and administration of specific antigens rather than crude microbial or tumor cell preparations is often a beneficial strategy to overcome the drawbacks described above.

Newly developed vaccines are rather based on selected target antigens, derived from an infectious microorganism, a tumor cell, an allergen or an auto-antigen. The target molecule may be administered as a purified protein or as a peptide(s), or may be expressed from plasmid DNA or a recombinant virus (Moingeon et al., 2002). Such molecular vaccines, beside of their advantages compared with conventional vaccines, have problems for use in medical application. The most important of these obstacles are briefly mentioned as follows.

Safety and ease of production are two of the particular advantages of peptide vaccines over the conventional vaccines (Purcell et al., 2007). However, problems include poor immunogenicity of the simple recombinant or synthetic peptide (Petrovsky and Aguilar, 2004; Purcell et al., 2007) and the need to potently stimulate T cells and elicit immunological memory. As yet, there are no human peptide-based vaccines on the market (Purcell et al., 2007; Li et al., 2014), this arises primarily from the difficulties associated with peptide stability and delivery, and the challenge posed by the diversity of human immunogenetics (Purcell et al., 2007).

For past two decades, nucleic acid vaccines, including DNA and RNA, had exhibited a great promise in immunotherapy for various diseases. Comparing with conventional vaccines, the nucleic acid vaccines have represented superiorities, such as good safety, specific to induce the immune response for the antigen of interest, induction of both $\mathrm{B}$ - and T-cell responses, relatively low cost of production, and ease of manufacturing. However, they have showed poor immunogenicity in clinic trials and have not yet been used in human as commercial products. The inherent barriers of the body, moreover, the poor stability and in vivo distribution of the naked form hinder 
the efficiency for nucleic acid vaccines (Yang et al., 2015).

Collectively, it is now clear that powerful strategiesparticularly an ideal delivery system- are needed to enhance the efficiency and specificity of the immunogenic vaccine components in the host body. These strategies are used to improve the vaccine components stability and immunogenicity in order to prompt an effective immune protection. Literature shows that the development of new vaccine delivery systems has become an intense research activity in the last years.

An ideal vaccine carrier should be able to efficiently present target antigens to the immune system (Moingeon et al., 2002; Souza et al., 2005) in a manner that would stimulate immune responses appropriate for protection against a specific disease (Moingeon et al., 2002), and it should be able to provide targeted delivery of vaccine components to certain sites of interest (Yang et al., 2015). Furthermore, the ideal vector system should be safe, and exhibit low intrinsic immunogenicity to allow for its readministration in order to boost relevant specific immune responses. The ease of its production on a large-scale should also be considered (Souza et al., 2005).

Viral vector is one of the approaches currently being used to deliver of vaccine components. These vectors have beneficial features over other available non-viral vectors. Nevertheless, the development of a novel and efficient non-viral delivery system is an important goal, because recombinant viruses still have a number of disadvantages as practical tools for medical application (Eguchi et al., 2001). Some aspects of viral vector application as a vaccine delivery system are discussed in more detailed latter. Recently, a great deal of attention has been focused on the bacteriophages for vaccine design purposes.

Bacteriophages (or phages) are bacterial viruses consisting of a DNA or RNA genome contained within a protein coat called capsid. Their growth and proliferation need a suitable prokaryotic host (Gao et al., 2010). Phages were discovered independently by Frederick Twort and Félix d'Hérelle, respectively, in 1915 and 1917 (Kaur et al., 2012).

Within the last years, bacteriophages found a variety of applications in biology and medicine due to their intrinsic properties such as high stability under a variety of harsh environmental conditions (including exposure to nucleolytic and proteolytic enzymes (Jepson and March, 2004; Lankes et al., 2007), desiccation, high temperatures and wide range of $\mathrm{pH}$ (Jepson and March, 2004)), the feasibility of large-scale production and manipulation. Other important advantages of bacteriophages that make them attractive are as follows; being a non-pathogenic and non-replicating vehicle in eukaryotic cells (Ulivieri et al., 2008), do not cause recombination events because of the nonexistence of eukaryote-related sequences (or homologous sequences) in their genome (Bakhshinejad and Sadeghizadeh, 2014), and do not carry antibiotic resistance genes (Clark and March, 2004).

Nowadays, the use of phages in different practical and therapeutic fields such as phage therapy (Bruttin and Brussow, 2005; Wright et al., 2009), phage display (Arap 2005), gene delivery (Yokoyama-Kobayashi and Kato, 1994; Khalaj-Kondori et al., 2011), bacterial typing and detection (Loessner et al., 1997; Neufeld et al., 2003; Schmelcher and Loessner, 2014), presents a confirmation of their robustness. Furthermore, because of the desired properties of phages, their ability also was investigated to develop a vehicle for immunogenic determinant delivery. To date, the phage based vaccines have been evaluated in areas of prevention and treatment of various diseases, and data showed that the immune response was primed. Thereby, this review attempts to have an insight into the important aspects in the application of phages as a vaccine carrier, including phage vaccine categories and their development, immunogenic properties of the phage particle, processing and presenting pathways of the phage carrying vaccine components in human cells. Furthermore, concerns and challenges regarding phage immunization for humans are addressed.

\section{Brief Review of Eukaryotic Virus-Based Vaccine Carriers}

Based on the observation that viral infection results in the presentation of virus-specific peptides in association with both MHC class I and MHC class II on the surface of infected cells, strategies have been designed to use viruses as immunization vehicles to elicit antigen-specific immune responses. In such approaches cDNA encoding one or several antigens, are inserted into the viral vector. The resulting recombinant viruses are used with the aim of the expression of the selected antigen(s) and their subsequent presentation to the immune system (Moingeon et al., 2002). Among the cells that can be transduced by these vectors, antigen-presenting cells (APCs) constitute an important subset that can be targeted by different viral vectors (Souza et al., 2005), these cells have a potential role in the immune response initiation and making the antigenic peptides (or proteins) accessible to the immune system (Abediankenari et al., 2010; Abediankenari et al., 2013).

Viral vectors offer important advantages when used as vaccines. These include high-level production of protein antigens directly within cells that are transduced, potential adjuvant effects of the viral delivery system itself and the possibility of efficient delivery of antigen directly to components of the immune system, such as antigen-presenting cells. A wide range of viruses have been extensively studied for their potential as recombinant vaccines and they all have the ability to induce humoral and cellular immune responses (Souza et al., 2005).

Nevertheless, the development of a novel and efficient delivery system for medical applications remains as an important goal and needs to be the focus of further investigation. Despite the many advantages which recombinant-viral vaccines can offer, pre-existing immunity (particularly neutralizing antibody) is a major obstacle of many viral-vectored vaccines, such as adenovirus serotype 5 or herpes simplex virus type 1 (HSV-1) (Saxena et al., 2013). In fact, a percentage of humans may be pre-exposed to the viral vectors and high levels of pre-existing immunity to the vector are presence in the human population (Souza et al., 2005). The existence of pre-existing immunity to the vector 
Phage Particles as Vaccine Delivery Vehicles: Concepts, Applications and Prospects

restricts delivery of the virus into cells, thereby effectively reduces the dose of the vectored antigen (Saxena et al., 2013). Indeed, pre-existing antibodies may eliminate recombinant virus particles before they are able to elicit an immune response (Souza et al., 2005). Several studies raised concerns about the use of the viral vectors in individuals with pre-existing immunity. For example, in a large-scale clinical trial of an adenoviruse serotype 5 (AdHu5)-based HIV-1 vaccine, the vaccines showed a lack of efficacy and tended to increase the risk of HIV1 infection in vaccine recipients who had pre-existing neutralizing antibodies to AdHu5. However, the inhibitory effect of pre-existing vector immunity can be avoided by several approaches (Saxena et al., 2013). Thus, the issue of prior exposure and pre-existing immunity to the virus in the human population needs to be considered in order to use the vector.

After vaccine administration, immunity to the viral vectors is also generated and this will play an important role limiting the subsequent use of the same vector for different vaccination purposes (Souza et al., 2005), presumably, because viruses will induce neutralizing antibody on the first dose, and this antibody, in subsequent doses, will limit the number of transduced cells, therefore this issue will restrict the responses (Saxena et al., 2013).

The potential pathogenicity of some viruses especially in infants and immunocompromised subjects and their oncogenic integration into the genome of host cells are other limitations in use of recombinant viral vaccines that may call into question the safety of viral based vaccines for general use (Souza et al., 2005; Bakhshinejad and Sadeghizadeh, 2014). The exploitation of virus-based carriers also imposes challenges including time-consuming, laborious, and expensive process of their production in eukaryotic cells (Bakhshinejad and Sadechizadeh, 2014).

These complications have triggered attempts to find novel vaccine delivery vehicles with desirable properties. Recently, a great deal of attention has been focused on the bacteriophages as a new category of potential vaccine carriers. Phage-based vaccines will be considered in detail in following paragraphs in order to establish a comprehensive view of this new class of vaccine.

\section{Phage-based Vaccines}

Phages do not replicate in eukaryotic hosts and act as inert particulate antigens metabolically. Being particulate antigens, phages are taken up and processed by antigenpresenting cells (APCs) (Gao et al., 2010). Thus, phage particle can target the vaccine to the APCs and this event makes them applicable as a vaccine delivery vehicle. The advantage of phage vaccines over naked DNA vaccines or peptide based vaccine is primarily the fact that virion protects the vaccine and makes it more stable for administration (especially in oral administration), storage and transport. Moreover, phage particles are themselves immunogenic and can act as a natural adjuvant (Manoutcharian et al., 1999; Clark and March, 2006; Gamage et al., 2009; Gao et al., 2011; Hashemi et al., 2012), therefore this method allows achieving better results at lower doses than standard vaccination. Previous studies showed that phage vaccines have the ability to induce both humoral and cellular immune responses (Clark and March, 2004; Gamage et al., 2009; Hayes et al., 2010).

Compared to viruses of eukaryotic organisms, phagebased vectors are much less likely to enter eukaryotic cells. The lack of intrinsic tropism of phages can dramatically lower the probability of non-targeted cargo delivery and its consequent adverse effects. For this reason, phage vectors show an inherent biological safety profile in mammalian organisms, making these vectors suitable for clinical uses. Hence, it is not necessary to modify phages for eliminating the possibility of nonselective tropism into mammalian cells; whereas for eukaryotic viruses homing to non-target cells is the cause of major concern, thus emphasizing the need to introduce changes in the viral structure to remove this concern (Bakhshinejad and Sadeghizadeh, 2014).

Although the safety of phage applications in humans was mentioned in many published works (Gamage et al, 2009; Bakhshinejad and Sadeghizadeh, 2014), the administration route of the phage vaccine must be considered because there is a risk that after application via the oral route, phages will infect intestinal flora (Bazan et al., 2012). In fact, phages need their target bacteria to grow, thus, their in vivo growth has the potential to release large quantities of endotoxin from lysed Gram-negative bacteria, with potential health concerns in human and animals (Clark and March., 2006). Albeit, this concern may not be related to all phages. Bruttin and Bru"ssow developed a study describing the oral administration of T4 phage to fifteen healthy human volunteers and the subsequent clinical and microbiological analysis. They concluded that oral phage application did not cause a decrease in total fecal Escherichia coli counts. In addition, no adverse events related to phage application were reported in this study (Bruttin and Bru“ssow, 2005). However, the safety of oral phages needs to be tested in more detail in order to use as a vector.

It should be mentioned that there are several strategies enhancing the oral administration of phage vaccines, for example, the use of virions with damaged tail fibers. These particles are incapable of infection but still can serve as a vaccine delivery vehicle (Bazan et al., 2012).

In use of phage for design of vaccine delivery vehicles, phage display vaccine and phage DNA vaccine can be constructed. Hybrid phage vaccine was also suggested (Gao et al., 2010). Developed phage vaccines are based on both icosahedral phages (such as lambda, T7, T4) and filamentous phages (such as Fd, F1, M13). The general consensus from published studies is that these vaccine vectors have the potential to be efficacious. The different categories of phage vaccines are described in the following sections.

\section{Phage Display Vaccine}

Phage display technique was first described by George Smith in 1985. In this technique, a foreign peptide or protein is expressed on the phage surface through transcriptional fusion with a coat protein gene (Smith 1985). Phage displayed library provides an opportunity 
for presenting of billions of pooled peptides on phage particles, then, these huge number of different peptides can be screened simultaneously for the desired affinity or activity (Bratkovic 2010). Nowadays, phage display technology are extensively used for various purposes in most fields of biological sciences such as epitope discovery (Cortese et al., 1994; Wang and Yu, 2004), epitope mapping and mimicking (Luzzago et al., 1993; Fack et al., 1997), identification of new receptor- ligands (Koivunen et al., 1999; Odermatt et al., 2001), making and isolation of antibodies (and their derivatives) with defined specificity and affinity (Winter et al., 1994).

Phage display also demonstrates a unique application of bacteriophages in modern vaccine design, in which phage display libraries can be screened with a specific antiserum to isolate novel protective peptides (antigens / mimotopes) (Clark and March, 2006), or phages can be reconstructed to display a specific protective antigen on their surface (Bazan et al., 2012). With carrying antigens on their surface, phages have been used to direct vaccination. Rather than generating a transcriptional fusion to a coat protein, vaccine antigens can also be artificially conjugated to the surface of phage particles, which allow for a wide range of antigens to be displayed (Clark and March, 2006). Indeed, bacteriophages could provide the possibility of being manipulated-both genetically and chemically- that improves their capabilities.

The first use of whole phage particles to elicit an immune response against displayed foreign peptides was reported in 1988. In this study, repeat regions of the circumsporozoite (CS) protein gene of the human malaria parasite Plasmodium falciparum were cloned into the pIII gene- expressing a minor coat protein- of the filamentous bacteriophage $\mathrm{f} 1$. These genetically engineered filamentous phages displayed the recombinant proteins on their surface. When recombinant phages were used for delivery of antigenic peptide, elicitation of responses to the fusion protein in one genetic background of mice and in rabbits was obtained (Cruz et al., 1988).

Within the past years, whole phage particles displaying antigenic proteins or mimotopes have been extensively exploited for the design of vaccine against various diseases such as cancers (Wu et al., 2002; Fang et al., 2005; Sartorius et al., 2008; Ren et al., 2009), and different microbial infections caused by viruses (Wan et al., 2001; Bahadir et al., 2011; Hashemi et al., 2012), parasites (Willis et al., 1993; Manoutcharian et al., 1999; Manoutcharian et al., 2004), bacteria (Jiang et al., 1997; Menéndez et al., 2011), fungi (Wang et al., 2006; Wang et al., 2014), and the protection against immune challenges was reported. Several studies are briefly described below.

The latent membrane protein 1 (LMP1) encoded by Epstein-Barr virus (EBV) has become a potential target in EBV-associated tumor immunotherapy due to its multiple biological effects. Several studies demonstrated that LMP1 is in fact a rather weak immunogen for both humoral and cellular immune responses (Meij et al., 2002a; Meij et al., 2002b). In a study by Gao and co-workers, the recombinant T7 phage displaying full-length LMP1 protein was investigated as an immunogen to immunize rats. The results of flow cytometry analysis indicated that the number of $\mathrm{CD} 3+, \mathrm{CD} 4+$, and CD8+ Tlymphocytes in rats immunized with T7-LMP1 phage increased. Reported results confirmed that both humoral and cellular immune responses were elicited (Gao et al., 2011).

Melanoma antigen genes (MAGE) are expressed in several types of human cancer but not in normal tissues, with the exception of male germ-line cells, and, to a lesser extent, placental throphoblast cells. Because both these cell types do not express MHC class I molecules and are incapable of presenting antigens to cytotoxic T-lymphocytes (CTLs), therefore, specific CD8+ T lymphocytes responses to antigens encoded by MAGE genes can be considered strictly tumor specific. Fang and co-workers displayed the tumor specific epitope of melanoma antigen A1 161-169 (MAGE-A1) on the major protein (pVIII) of the filamentous bacteriophage (fd). The mice were immunized with modified phage particles, and then cellular immune responses of mice were examined. The results showed that these phage particles prevented tumor growth and was effective in prolonging survival of tumor-bearing mice. Also, immunization primed a MAGE-A1 epitope specific CTL response, elicited DTH (delayed-type hypersensitivity) response and enhanced the NK cells activity (Fang et al., 2005).

Ectodomain of influenza A virus M2 protein (M2e) is a candidate vaccine antigen for development of a universal flu vaccine. However, M2e has a poor immunogenicity during a natural influenza A virus infection and induces very low titers of M2e-specific antibodies in humans, presumably due to its limited expression on virions. Hashemi et al. developed a universal influenza vaccine based on $\mathrm{T} 7$ phage particles displaying a high density of M2e peptide (T7-M2e). The immunogenicity of the T7-M2e particles was assessed in mice. Results showed that immunization with M2e -when displayed densely on the surface of T7 particles- successfully elicited both IgG1/IgG2a and $\mathrm{T}$ cell responses after three subcutaneous immunizations. Moreover, a high level of protection (66\% and $83 \%$ survival rates) was achieved against lethal challenge with two different mouse-adapted subtypes of influenza A virus (PR8 and X47 viruses). T7-M2eimmunized mice had significantly reduced viral load in their lungs compared to controls. By contrast, M2e peptide only triggered a minimal response when simply mixed with wild type T7 particles, this response not strong enough to protect mice against a lethal virus infection. This observation confirmed the key role of particulate delivery and multivalent arrangement of M2e peptide on the surface of nanoparticles in triggering a strong activation signal via crosslinking of B cell receptors and subsequent antibody production (Hashemi et al., 2012).

It is worthy to note that a phage expressing a mixture of wild type and fusion coat protein is introduced as "hybrid" phage in literature. Construction of doubledisplay hybrid virions was also reported, in which two different epitopes from a pathogen or an effector sequence and a targeting sequence could be displayed simultaneously on the same capsid (Malik and Perham, 1997). A double hybrid bacteriophage displaying an immunodominant B- and T-helper cell epitope pep23, KDSWTVNDIQKLVGK, corresponding to residues 249- 
263 of the reverse transcriptase (Rtase) of HIV-1, together with a CTL epitope RT2, ILKEPVHGV, corresponding to residues 309-317 from the same Rtase, was capable of eliciting a helper-dependent anti-HIV-1 CTL response (De Berardinis et al., 2000).

Alternatively, phages displaying peptide libraries can be screened to isolate novel protective epitope-mimics, so-called mimotopes- peptides that mimic the secondary structure and antigenic properties of a protective carbohydrate, protein, or lipid (Clark and March, 2006; Knittelfelder et al., 2009). Mimotopes may not have similarity to any linear sequence (or primary structure) of the antigen and may represent conformational dependent interaction of the epitope with the antibody (Pande et al., 2010). In fact, mimotopes mimic an epitope by their biochemical and electrostatic properties, and not necessarily by sequence homology (Knittelfelder et al., 2009). These peptides can lead to the development of a new class of vaccines, based on 'traces' left by the pathogen in the immune response of patients. Thus, using sera from immunized patients, it is possible to select phage displayed peptides that mimic the original antigen's characteristic of binding to specific antibodies. Response to the mimotopes is generally much weaker, but comparable to that obtained by immunizing with the original antigen itself (Delmastro et al., 1997). In a study by Delmastro et al. selected human sera was used to identify mimotopes of human HBV surface antigen and of human HCV peptides displayed on bacteriophages. They showed that oral administration of phages displaying HBV surface antigen and $\mathrm{HCV}$ peptide mimics (mimotopes) can induce antibodies specific for the original viral antigen. These findings indicated that phage displaying selected mimotopes could be useful for the development of orally effective vaccines (Delmastro et al., 1997).

Although effective plain or conjugated capsular polysaccharide-based vaccines are available against serogroups A, C, Y and W135, there is still no vaccine capable of eliciting broadly protective antibodies to most Neisseria meningitidis serogroup B strains. In a study by Menéndez et al. identification and characterization of phage-displayed peptide mimetics of $N$. meningitidis serogroup B capsular polysaccharide was performed. They screened a phage-displayed peptide library using a bactericidal and protective $\operatorname{IgG} 2$ a monoclonal antibody (13D9). In order to examine the capacity for immunogenic mimicry of the isolated peptides, mice were immunized directly with the phage-displayed peptides. Induction of anti-peptide $\mathrm{IgG}$ antibodies, mainly $\operatorname{IgG} 2 \mathrm{a}$ for three of the peptides, bactericidal and protective antibody levels for one of them was shown. Thus, these peptides could be considered as vaccine candidates against serogroup B meningococcal strains (Menéndez et al., 2011).

Two ways in which phage have been used to deliver vaccines are showed as Figure 1.

\section{Phage DNA Vaccines}

Nucleic acid vaccines are used to deliver genetic materials encoding protective antigens into host cells. They can directly express antigen protein in suit and

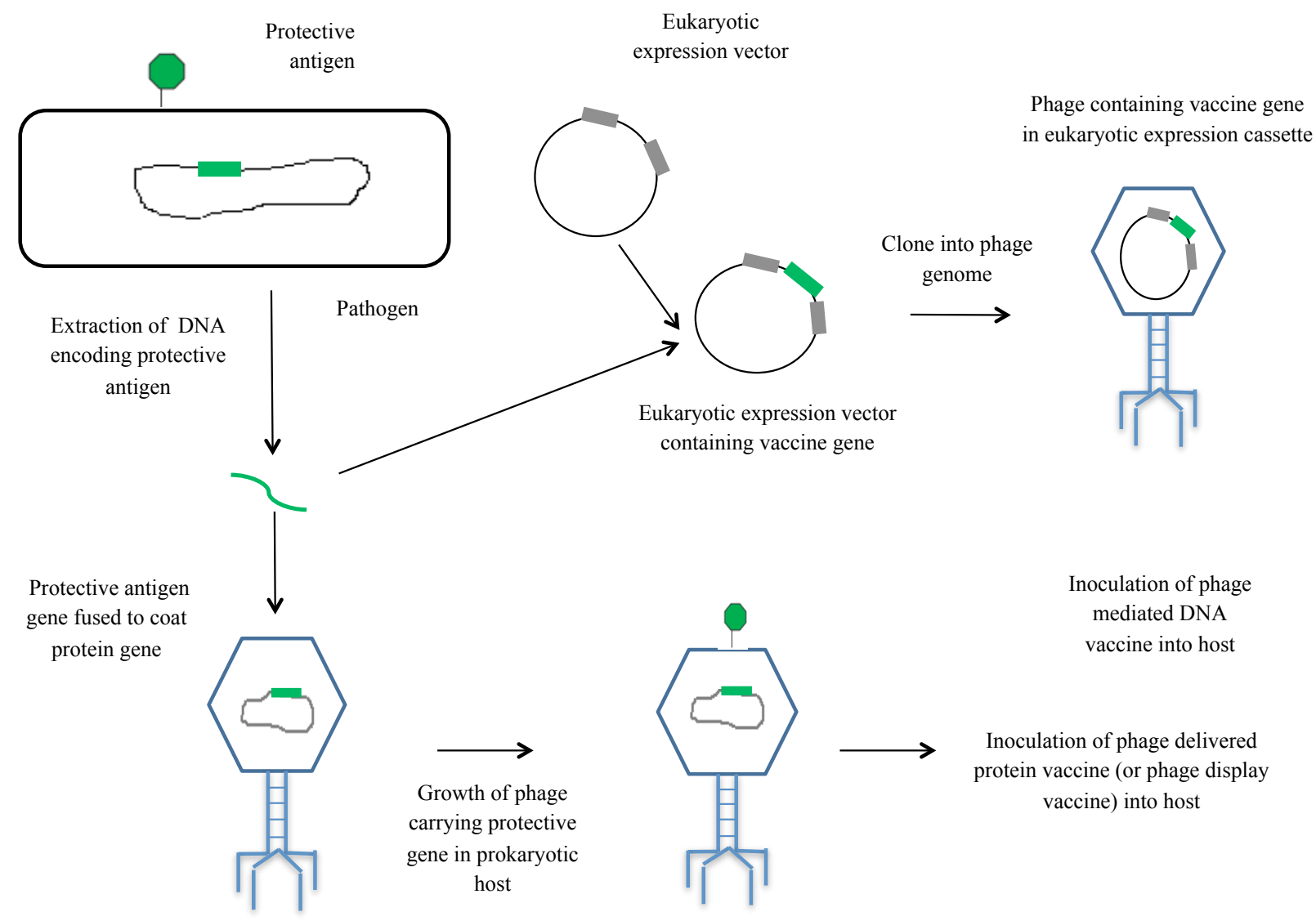

Figure 1. A Schematic Diagram of Two Vaccine Delivery Methods Via Phages, Namely Phage Display Vaccine And Phage DNA Vaccine 
initiate immune responses of the host to protect against following challenge and disease therapy. The development of a nucleic acid delivery system is a promising strategy for safe and effective immune protection and therapy, since delivery vectors could improve nucleic acid stability and immunogenicity, and also target delivery to the cells of interest- APCs (Yang et al., 2015).

In phage DNA vaccine, the gene encoding the vaccine antigen, under the control of a eukaryotic expression cassette, is cloned into the phage genome. Then, the vaccine gene is packed within phage virion, therefore, whole phage particles are used to deliver a DNA vaccine expression cassette into the host (Figure 1). Being particulate antigens, bacteriophages are taken up by APCs and cloned inserts are expressed by these cells (Clark and March, 2004; March et al., 2004). Thus, phage represents an extremely simple and effective technology for targeting DNA vaccines to APCs (March et al., 2004). Indeed, phage DNA vaccine procedures use phages without surface modifications to deliver DNA vaccines.

It is worthy to note that the eukaryotic cassette is a part of vector DNA which contains three main sequences: promoter, ORF (open reading frame) and 3'untranslated region often with polyadenylation site (Bazan et al., 2012). This construction allows the proper gene expression and correct folding of the gene delivered by bacterial viruses in eukaryotic host cells.

Compared to standard naked DNA vaccine technology, this system, namely recombinant bacteriophage particle containing DNA vaccines, offers several advantages. Vaccine nucleic acid is contained within a stable matrix that can be targeted to the APCs and protected from nuclease degradation (Clark and March, 2004). Expression constructs of up to $15-20 \mathrm{~kb}$ in size can be used in a standard lambda $(\lambda)$ bacteriophage vector (50 $\mathrm{kb}$ ), raising the possibility that multiple vaccines can be delivered within a single phage or that large eukaryotic intron-containing genes can be used directly as nucleic acid vaccines (Clark and March, 2004). This feature gives $\lambda$ phage a tremendous advantage over filamentous phages (fd, f1, M13) that have a smaller genome size $(6.4 \mathrm{~kb})$.

To date, few studies have been focused on the use of the phage as a DNA vaccine delivery vehicle. For the first time, in a study by Clark and March, whole $\lambda$ bacteriophage particles containing reporter gene (green fluorescent protein (GFP)) under the control of the cytomegalovirus promoter $\left(\mathrm{P}_{\mathrm{CMV}}\right)$, have been used as delivery vehicles for investigation of nucleic acid immunization. When peritoneal macrophages were incubated with whole $\lambda$ phage particles, GFP antigen was detected on the macrophage surface $8 \mathrm{~h}$ later. These results suggested that direct targeting of APCs by bacteriophage vaccines may occur, and cloned inserts were expressed by APCs (Clark and March, 2004; March et al., 2004). Clinical outcome of chronic Hepatitis B virus infection is linked to immune response. The disease may develop to liver carcinoma (Abediankenari et al., 2011), thus, methods should be considered to overcome the virus infection. In another experiment, following intramuscular injection of mice and rabbits with $\lambda$ phage particles containing hepatitis B surface antigen (HBs Ag) gene, anti$\mathrm{HBs}$ Ag responses were detected. Their study showed that bacteriophage-mediated DNA vaccination consistently gave better antibody responses when compared to naked DNA, although plasmid copy numbers were 50-200 times higher than phage copy numbers (Clark and March, 2004; March et al., 2004).

Later, different small and large animal models have been vaccinated by phage DNA vaccines and the results demonstrated a long-lasting and significantly higher antibody response compared to naked DNA vaccine or even purified recombinant protein (Kaur et al., 2012). Some of these reports are described in the following paragraphs.

Human papillomaviruses (HPV) have been identified as the etiological agent of cervical carcinomas. In a study by Ghaemi and co-workers, a recombinant $\lambda$-ZAP E7 phage vaccine was developed by inserting the HPV16 E7 gene, under the control of the cytomegalovirus promoter, into the $\lambda$ - ZAP vector. Lambda-ZAP E7 phages were employed to immunize mice against challenge with E7expressing tumor cell line (TC-1). The tumor-bearing mice indicated a significant inhibition of tumor growth after recombinant phage injections. Released lactate dehydrogenase, interferon- $\gamma$ and granzyme B from spleen cells, and lymphocyte proliferation of spleen cells demonstrated the enhancement of cell-mediated immunity. Results suggested that the recombinant phages can be used as effective vaccines for inducing E7-specific protective immune responses (Ghaemi et al., 2010).

Chlamydia abortus is an obligate intracellular pathogen that causes abortion in pigs and poses a zoonotic risk in pregnant women. Although attenuated and inactivated vaccines are available, they do not provide complete protection in animals. The major outer membrane protein (MOMP) of the C. abortus EB (elementary body) was considered to be an important target for generating protective immune responses against C. abortus infections. Ou et al. evaluated an ompA-based phage-mediated DNA vaccine against $C$. abortus in piglet. Groups of piglets were immunized intramuscularly with the phage-MOMP vaccine ( $\lambda$-MOMP) or a commercial live-attenuated vaccine (1B vaccine). The results indicated that immunization with the $\lambda$-MOMP vaccine elicited significantly higher antigen-specific serum IgG antibody and lymphocyte proliferative responses compared to those immunized with the $1 \mathrm{~B}$ vaccine or control $(\mathrm{Ou}$ et al., 2013).

\section{Hybrid Phage Vaccines}

The hybrid phage vaccine results from a combination of phage display and phage DNA vaccine strategies. The hybrid phage vaccine delivers both protein and DNA vaccine in one construct. This model allows to display not only antigen but also a targeting molecule, aimed to increase uptake of the phage by specific cell types (Gao et al., 2010). Previous studies demonstrated that display of receptor-binding peptides in a multivalent fashion (or multiple copies) is required for efficient phage internalization (Ivanenkov et al., 1999). 
In fact, this method suggests possible modifications for improving phage-based DNA vaccine delivery, thus, permits both cell targeting and delivery of genetic materials encoding protective antigens. However, it seems that the rationale for using phages as hybrid vaccine is similar to that for using phages for targeted gene delivery applications.

Although previous literature (Gao et al., 2010) considered the third class of phage vaccine as a hypothesis, it seems that several previous experiments can help us to understand the concept of the hybrid phage vaccine.

$\mathrm{A}_{\mathrm{v}} \beta_{3}$ integrin is known to play a role in the binding and /or internalization of a number of mammalian viruses including adenoviruses and some hantaviruses. In the past, this integrin successfully used to enhance the targeting of modified virus vectors to dendritic cells, which are known to express the receptor. In a study by Lankes and co-workers, lambda phage vectors, displaying a peptide known to bind to the $\alpha_{v} \beta_{3}$ integrin receptor, were found to be efficiently internalized into mammalian cells expressing this integrin. The modified lambda phage vector mediated an enhanced efficiency of gene transfer in $\alpha_{\mathrm{v}} \beta_{3}$-positive cells in comparison to identical host cells that did not express this receptor. Consistent with the results of these in vitro experiments, in vivo studies suggested that targeting of phage to integrin receptors can indeed increase the efficiency of phage-mediated gene transfer. Interestingly, these researchers found a 100 -fold increase in the efficiency of phage internalization into $\alpha_{\mathrm{v}} \beta_{3}$-positive cells compared to control cells, but only about a 3 -fold increase in the level of phage-mediated gene expression. This result suggested that only a small fraction of the internalized phage particles were able to effectively deliver their genetic payload (Lankes et al., 2007). This event might be due to the mammalian intracellular barriers such as endosomal, lysosomal, proteasomal and nuclear membrane, for phage-mediated gene expression. As a consequence, the efficiency of expression is less than the efficiency of internalization. These barriers impose limitations on the expression of the transferred gene by phages (Bakhshinejad and Sadeghizadeh, 2014). However, the genetic flexibility of bacteriophages can be exploited to manipulate their surface features for adapting them for efficient gene expression in human cells.

In this regards, several strategies have been suggested to reach a higher rate of phage-mediated gene expression in mammalian cells. A number of these strategies are based on mimicking the methods used by eukaryotic viruses including the exploitation of endosome-disrupting factors and NLSs (nuclear localization signals). For example, the plasma membrane of mammalian cells is one of the tight barriers against gene transfer by synthetic delivery systems. In a study (Eguchi et al., 2001), recombinant $\lambda$ phage particles displaying Tat peptide- the transduction domain of human immunodeficiency virus (HIV) type 1 Tat protein- on their surfaces and carrying mammalian marker genes as part of their genomes (Tat-phage) was constructed. When animal cells were exposed to Tatphage, significant expression of phage marker genes was induced. Indeed, Tat peptide greatly promoted gene transfer via membrane destabilization (Eguchi et al., 2001).

\section{Immunogenic properties of phage particles}

Phage particles are naturally immunostimulatory. An interesting research on the phage as an immunogenic carrier was done by van Houten and colleagues. In this study, mice were immunized with ${ }^{(i)}$ synthetic B2.1 peptide conjugated to f1.k phage (f1.k/B2.1); (ii) phagedisplayed recombinant B2.1 peptide (B2.1 phage); or (iii) synthetic B2.1 peptide conjugated to ovalbumin (OVA/ B2.1) -a traditional carrier. Then, the serum antibody titers were measured. Result showed that all immunogens elicited anti- B2.1 peptide antibody titers and the OVA/ B2.1 conjugate was the best immunogen, since it elicited stronger anti-peptide antibody responses compared to the two phage immunogens. In this study, antibody response focused against the B2.1 peptide was also calculated as the ratio of the anti-peptide antibody response to the anti-carrier antibody response, reflecting the relative strength of antibody response against the peptide. They concluded that higher peptide-to-carrier antibody ratios were elicited by the $\mathrm{f} 1 . \mathrm{K} / \mathrm{B} 2.1$ conjugate, indicating that phage, as a carrier, appears better at focusing the antibody response against peptide than the traditional carrier OVA, perhaps due to OVA's more extensive set of BCEs (B- cell epitopes) compared to the restricted BCEs on the phage (Houten et al., 2006).

Indeed, the antibody response to the filamentous phage is directed exclusively against restricted BCEs on pVIII and pIII. The antibody response to pVIII is restricted to 12 residues at the $\mathrm{N}$-terminus, thus, there may be a limited number of B-cell clones that could elicit antibodies against pVIII. In the case of pIII, whose 200 - residue outer domains are immunogenic, there may be more epitope diversity in the exposed $\mathrm{N} 1$ and $\mathrm{N} 2$ domains; however, this protein is present in limited amounts (3-5 copies/phage), which may reduce its immunogenicity. These properties suggest that phage is proficient carriers for synthetic and recombinant peptides in situations requiring a focused immune response. But, a disadvantage of using the filamentous phages is the relatively weak T-cell help that they can provide. The larger minor coat proteins on phage, pIII (42.5 kDa), may contain more TCEs than those found on pVIII $(5.5 \mathrm{kDa})$, however, these proteins are present in proportionally small amounts (Houten et al., 2006).

Furthermore, it was proposed that phage can be engineered to enhance and focus the immune response against a peptide (synthetic or recombinant), for example, by the addition of foreign CD4+T-cell epitopes to pIII or pVIII or a further reduction of its BCEs. In another work, the effect of reducing the phage surface complexity on the antibody response against peptides that were either displayed as recombinant fusions to the phage coat or were chemically conjugated to it, was investigated. The results suggested that focusing of the antibody response against synthetic peptides can be improved by decreasing the antigenic complexity of the phage surface, but this effect depends on peptide's copy number (or antigen valency), its inherent immunogenicity, and the manner in which the peptide is presented on the phage surface 
(Houten et al., 2010).

The N-terminal domain of filamentous bacteriophage coat protein III (DI) molecule was used in a genetic vaccination as an adjuvant by Cuesta and co-workers. In this study, a naked DNA plasmid vaccine was constructed by fusing non-immunogenic L36 $\mathrm{scFv}$ - an antilaminin $\mathrm{scFv}$ fragment that inhibits angiogenesis both in vitro and in vivo- to the first 80 translated non-leader sequence codons of phage gene III. In mice, the immunization with the naked DNA plasmid vaccine encoding scFv-DI fusion efficiently induced a Th1 immune response, characterized by a dominant L36-specific IgG2a humoral response and high levels of IFN- $\gamma$ produced by splenic T cells. Immunity was not elicited by naked DNA plasmid encoding nonfused scFv (Cuesta et al., 2006).

Previous studies showed that phage particles do not require an adjuvant for application as a vaccine carrier (Willis et al., 1993). This may be, in part, due to the particulate nature of the phage virion as well as to the presence of significant amounts of E.coli LPS (lipopolysaccharide) associated with purified phage preparations. However, the effect of LPS can be removed by treating the phage with detergent after growth in bacteria (Irving et al., 2001). Some studies also showed that a better immune response induction occurs in presence of an adjuvant. For example, in a study, M2e peptide was displayed on the surface of $\mathrm{T} 7$ particles for developing a protective influenza vaccine (T7-M2e). After removal of the bacterial LPS, the vaccine immunogenicity was assessed in the presence or absence of a strong adjuvant in mice. T7-M2e particles without any additional adjuvant successfully elicited both IgG1/IgG2a and T cell responses in mice that were similar in amounts to those elicited by M2e peptide administered in Freund's adjuvant. However, when T7-M2e particles were emulsified in Freund's adjuvant, significantly higher M2e-specific IgG titers especially of Th1-induced IgG2a isotype and stronger $\mathrm{T}$ cell response (IFN- $\gamma$ secretion in splenocytes) were obtained and the survival rate of mice was improved against a lethal infection with both PR8 and X47 viruses (Hashemi et al., 2012).

Despite the vast amount of data showing the potential of phage as vaccine carrier, there have been very few studies describing the effect of carrier specific immune response on the induction of vaccine antigen specific immune response. However, according to the a few available documents, there are conflicting reports indicating that pre-existing phage carrier specific immunity could enhance (Clark and March, 2004; March et al., 2004; Lankes et al., 2007), have no effect (Hashemi et al., 2012; Ou et al., 2013) or suppress (Gamage et al., 2009) the specific immune response against the target vaccine antigen.

IgG responses against $\mathrm{T} 7$ phage capsomers were induced following vaccination with T7 displaying M2e peptide (T7-M2e). However, induction of anti-M2e IgG response was not inhibited in mice immunized three times with T7-M2e particles, because high anti-M2e IgG titers were detected in sera despite high levels of anti-T7 carrier IgG antibodies. In this study, a relatively high dose of T7-M2e particles $\left(10^{9} \mathrm{pfu} /\right.$ mouse $)$ carrying a high copy of M2e peptide per particle was used that probably inhibited the epitope suppression by anti-T7 IgG. This reported data suggested that pre-existing anti-T7 phage antibodies would not interfere with the rise of anti-M2e peptide antibodies in booster administrations (Hashemi et al., 2012).

Lambda phage particles displaying PCV2 (porcine Circovirus 2) capsid protein epitopes was examined to develop a potential PCV2 vaccine candidate and was shown to be immunogenic in pigs. The vaccinated pigs showed significant anti-PCV2 immune response at primary vaccination, which plateaued following the second vaccination and slightly decreased after the third vaccination. The decline in the anti-PCV2 immune response following the third immunization might be due to the high level of carrier specific immunity at this stage of the experiment that contributed to a suppressor effect (Gamage et al., 2009).

$\mathrm{Ou}$ et al. evaluated an ompA-based phage DNA vaccine ( $\lambda$-MOMP) against $C$. abortus in piglet. Antibody responses against the $\lambda$-phage coat proteins were observed in the $\lambda$-MOMP vaccinated group. However, these antibodies did not seem to interfere with immune responses to MOMP, as high levels of MOMP-specific antibody and T-cell responses were induced in the piglets following vaccination with the $\lambda$-MOMP vaccine (Ou et al., 2013).

March and colleagues observed a high antibody response against $\lambda$ phage coat proteins in rabbits vaccinated with HBs antigen-encoding phage particles $(\lambda$-HBsAg as a $\lambda$-phage DNA vaccine) following the first vaccination, suggesting that the high anti-phage response did not inhibit the efficiency of subsequent phage vaccinations. In addition, they reported a high level immune response against the reporter/vaccine component following multiple intramuscular injections of the $\lambda$-HBsAg vaccine (Clark and March, 2004; March et al., 2004). Consistent with the latter study, Lankes and coworkers found that the presence of pre-existing phage-specific antibodies was associated with an increase in phage-mediated reporter gene expression (Lankes et al., 2007).

Collectively, the different observations can be attributed to the nature of the two types of vaccines, namely, phage display vaccine and phage DNA vaccine. In use of a candidate display vaccine, the amount of administered antigen will degrade over the time (Gamage et al., 2009). But, the high anti-phage response may enhance the efficiency of the delivery system carrying a DNA vaccine, since the formation of immune complexes should more effectively target APCs following boosting, where the DNA vaccine gene is expressed.(Clark and March, 2004; March et al., 2004). In fact, expression of the antigen from eukaryotic promoter contained in a phage DNA insertion cassette might overcome an anti-phage suppressive effect (Gamage et al., 2009). Furthermore, Hashemi et al. suggested that using of a high dose of phage particles displaying vaccine peptide and carrying a high copy of vaccine peptide can probably inhibit the epitope suppression by anti-phage immune response (Hashemi et al., 2012). 
Uptake Mechanism and Intracellular Fate of Phage Particles

The best-studied endocytosis mechanisms of eukaryotic cells for large particle uptake are clathrinmediated endocytosis, caveolae-mediated endocytosis, and macropinocytosis (Kim et al., 2012).

In order to address the mechanism of phage internalization into mammalian cells, and to test whether phagocytosis was required for this process, an examination was performed using $\lambda$ phage particles encoding firefly luciferase (luc) as a reporter gene. Mice were pretreated with clodronate-containing liposomes. This resulted in extensive depletion of phagocytic cells but did not significantly decrease phage-mediated gene expression (although a non-significant trend towards reduced luc expression was detected). The simplest explanation for these findings is that phagocytosis is responsible for a portion, but not the majority, of phage-mediated gene transfer. Thus, it is likely that other mechanisms may contribute to the uptake of phage particles. These include macropinocytosis (Lankes et al.,2007) - an actin-dependent endocytotic mechanism that involves internalization of relatively large ( $>1 \mu \mathrm{m}$ ) patches of membrane (Kim et al., 2012). Cell types capable of performing macropinocytosis include epithelial cells and keratinocytes, which would not be depleted by exposure to clodronate, as well as CD8+ interdigitating DC, which are known to be clodronateresistant, unlike marginal DC and macrophages (Lankes et al., 2007). Internalization of bacteriophage virions through macropinocytosis is suggested in other works (Gaubin et al., 2003), but, further studies will be needed to determine whether macropinocytosis indeed contributes to phage internalization into mammalian cells (Lankes et al., 2007).

It seems that displayed cell-penetrating peptides on the phage surface can determine the internalization mechanism (or endocytotic pathway). Kim et al. investigated the internalization mechanism of filamentous M13 phages displaying the cell-penetrating 3D8 VL transbody (3D8 VL-M13) or TAT peptide (TAT-M13) on the surface via fusion with the minor coat protein $\mathrm{pIII}$ (g3p). Recombinant 3D8 VL-M13 and TAT-M13 phages were efficiently internalized into living mammalian cells via physiological and energy-dependent endocytosis. Further studies revealed that 3D8 VL-M13 was internalized principally by caveolae-mediated endocytosis via interaction with heparan sulfate proteoglycans (HSPG) as cell surface receptors, whereas TAT-M13 was internalized by clathrin- and caveolae-mediated endocytosis utilizing chondroitin sulfate proteoglycans (CSPG) as cell surface receptors, suggesting that phage internalization occurs through specific cell surface receptors rather than nonspecific transcytotic pathways. Also, they found that macropinocytosis was not involved in 3D8 VL-M13 and TAT-M13 internalization (Kim et al., 2012).

Gaubin and co-workers investigated the processing mechanisms of filamentous bacteriophage Fd in EpsteinBarr virus-transformed B cell line (EBV-B) as antigen presenting cell. The major coat protein (pVIII) in intact virions was labeled with fluorescein isothiocyanate
(FITC), and its localization in various intracellular compartments was followed using confocal microscopy. They observed that fluorescent virions were efficiently internalized by EBV-B lymphocytes, where they reach the early and late endosomes and are degraded. Fluorescent protein fragments were located in lysosomal and MHC (major histocompatibility complex) class II compartments; they were also found in endoplasmic reticulum (ER), for peptide loading onto MHC class II and class I molecules, respectively. This suggested that filamentous bacteriophage ( $\mathrm{fd}$ ) virions are capable of being taken up and processed efficiently by mechanism of MHC class I and II pathways, even in nonprofessional antigen presenting cells (Gaubin et al., 2003).

Cross-presentation of phage particle antigen in MHC class II and ER marker -positive compartments was also found in other studies (Wan et al., 2005). These studies suggested that phages could represent the means by which exogenous antigen can reach the MHC class I pathway (Gaubin et al., 2003; Wan et al., 2005). MHC class I- peptide complexes are able to stimulate a CTL response, and the CTL activity plays a key role in the host defense against tumours and viral infections (Cuesta et al., 2006). In fact, one of the major limitations of vaccines containing non-living components is their inability to reach the MHC class I compartment and to stimulate a CTL response. Peptides recognized by CD8+ CTLs usually- but not always- result from the processing of endogenous intracellular proteins, and are presented by MHC class I molecules (Gaubin et al., 2003). The ability of bacteriophages to enter both MHCI and MHCII pathways of antigen processing and presentation may be exploitable in the construction of safe subunit vaccines (Gaubin et al., 2003; Ulivieri et al., 2008).

\section{Conclusions}

In conclusion, Over the recent years, considerable efforts have been devoted to develop ideal vaccine carriers for application in the treatment or prevention of diseases. It is clear that an ideal vaccine delivery system that is applicable for humans, should be safe and have no side effects. It should enable efficient presentation of vaccine components to the immune system and effectively stimulate the enhanced cellular and humoral immune responses. This system must also meet criteria that enable its large scale production.

Phages, because of their desired features, provide great promise for the development of a vaccine delivery system, but, their application deserves further studies. Apart from the knowledge that phage particles are safe in eukaryotes, further substantial evidence should be acquired in the clinical trials and particularly in oral administration. Conduct of clinical trials, notably involving individuals who are positive for anti- phage antibodies, is also needed to investigate the effectiveness of phage display vaccines and their immunostimulation in hosts. Furthermore, it seems that using of several strategies such as endosomedisrupting factors and NLSs (to facilitate DNA entry into nucleus of APCs), one can improve the expression of phage-mediated DNA vaccine, and in consequence, 
the immune response induction in mammalian cells. Also, further investigation is required to gain a better understanding of the immunostimulative mechanism of phage-based vaccines. Certainly, considering the points mentioned above, further research will accelerate the development and wide use of phage-based vaccines for clinical application.

\section{References}

Abediankenari S, Ghasemi M, Nasehi MM, Abedi S, Hosseini $\mathrm{V}$ (2011). Determination of trace elements in patients with chronic Hepatitis B. Acta Medica Iranica, 49, 667-669.

Abediankenari S, Janbabaei Mollae G, Ghasemi M, et al (2013). Vaccination of diffuse large B-cell lymphoma patients with antigen-primed dendritic cells. Acta Medica Iranica, 51, 284-288.

Abediankenari S, Yousefzadeh Y, Azadeh H, Vahedi M (2010). Comparision of several maturation inducing factors in dendritic cell differentiation. Iran J Immunol, 7, 83-87.

Arap MA (2005). Phage display technology - Applications and innovations. Genet Mol Biol, 1, 1-9.

Bahadir AO, Balcioglu BK, Uzyol KS, et al (2011). Phage displayed HBV core antigen with immunogenic activity. Appl Biochem Biotechnol, 165, 1437-47.

Bakhshinejad B, Sadeghizadeh M (2014). Bacteriophages as vehicles for gene delivery into mammalian cells: prospects and problems. Expert Opin Drug deliv, 11.

Bazan J, Całkosiński I, Gamian A (2012). Phage display-A powerful technique for immunotherapy. Hum Vaccin Immunother, 8, 1829-35.

Bratkovic T (2010). Progress in phage display: evolution of the technique and its applications. Cell Mol Life Sci, 67, 749-67.

Bruttin A, Bru"ssow H (2005). Human volunteers receiving Escherichia coli phage T4 orally: a safety test of phage therapy. Antimicrob Agents Chemother, 49, 2874-8.

Clark JR, March JB (2004). Bacteriophage-mediated nucleic acid immunization. FEMS Immunol Med Microbiol, 40, 21-26.

Clark JR, March JB (2006). Bacteriophages and biotechnology: vaccines, gene therapy and antibacterials. Trends Biotechnol, 24, 212-8.

Cortese R, Felici F, Galfre G, et al (1994). Epitope discovery using peptide libraries displayed on phage. Trends Biotechnol, 12, 262-7.

Cuesta A M, Suárez E, Larsen M, et al (2006). Enhancement of DNA vaccine potency through linkage of antigen to filamentous bacteriophage coat protein III domain I. Immunology, 117, 502-6.

De Berardinis P, Sartorius R, Fanutti C, et al (2000). Phage display of peptide epitopes from HIV-1 elicits strong cytolytic responses. Nat Biotechnol, 18, 873-6.

de la Cruz VF, Lal AA, McCutchan TF (1988). Immumogenicity and epitope mapping of foreign sequences via genetically engineered filamentous phage. J Biol Chem, 263, 4318-22.

Delmastro P, Meola A, Monaci P, Cortese R, Galfrè G (1997). Immunogenicity of filamentous phage displaying peptide mimotopes after oral administration. Vaccine, 15, 1276-85.

Eguchi A, Akuta T, Okuyama H, et al (2001). Protein transduction domain of HIV-1 Tat protein promotes efficient delivery of DNA into mammalian cells. J Biol Chem, 276, 26204-10.

Fack F, Hugle-Dorr B, Song D, et al (1997). Epitope mapping by phage display: random versus gene-fragment libraries. $J$ Immunol Methods, 206, 43-52.

Fang J, Wang G, Yang Q, et al (2005). The potential of phage display virions expressing malignant tumor specific antigen MAGE-A1 epitope in murine model. Vaccine, 23, 4860-6.
Gamage LNA, Ellis J, Hayes S, et al (2009). Immunogenicity of bacteriophage lambda particles displaying porcine Circovirus 2 (PCV2) capsid protein epitopes. Vaccine, 27, 6595-604.

Gao J, Liu Z, Huang M, Li X, Wang Z (2011). T7 phage displaying latent membrane protein 1 of Epstein-Barr virus elicits humoral and cellular immune responses in rats. Acta Virol, 55, 117-21.

Gao J, Wang Y, Liu Z. Wang Z (2010). Phage display and its application in vaccine design. Ann Microbiol, 60, 13-19.

Gaubin M, Fanutti C, Mishal Z, et al (2003). Processing of filamentous bacteriophage virions in antigen-presenting cells targets both HLA class I and class II peptide loading compartments. DNA Cell Biol, 22, 11-18.

Ghaemi A, Soleimanjehi H, Gill P, et al (2010). Recombinant $\lambda$-phage nanobioparticles for tumor therapy in mice models. Genet Vaccines Ther, 8(3).

Hashemi H, Pouyanfard S, Bandehpour M, et al (2012). Immunization with M2e-displaying $\mathrm{T} 7$ bacteriophage nanoparticles protects against influenza A virus vhallenge. PLOS ONE, 7, 1-11.

Hayes S, Gamage NA, Hayes C (2010). Dual expression system for assembling phage lambda display particle (LDP) vaccine to porcine Circovirus 2 (PCV2). Vaccine, 28, 6789-99.

Irving MB, Pan O, Scott JK (2001). Random-peptide libraries and antigen-fragment libraries for epitope mapping and the development of vaccines and diagnostics. Curr Opin Chem Biol, 5, 314-24.

Ivanenkov VV, Felici F, Menon AG (1999). Uptake and intracellular fate of phage display vectors in mammalian cells. Biochim et Biophys Acta, 1448, 450-62.

Jepson CD, March JB (2004). Bacteriophage lambda is a highly stable DNA vaccine delivery vehicle. Vaccine, 22, 2413-19.

Jiang J, Abu-Shilbayeh L, Rao BV (1997). Display of a PorA peptide from Neisseria meningitidis on the bacteriophage T4 capsid surface. Infect Immun, 65, 4770-77.

Kaur T, Nafissi N, Wasfi O, et al (2012). Immunocompatibility of Bacteriophages as Nanomedicines. J Nanotechnol, 1-13.

Khalaj-Kondori M, Sadeghizadeh M, Behmanesh M, Saggio I, Monaci P (2011). Chemical coupling as a potent strategy for preparation of targeted bacteriophage-derived gene nanocarriers into eukaryotic cells. J Gene Med, 13, 622-31.

Kim A, Shin TH, Shin SM, et al (2012). Cellular internalization mechanism and intracellular trafficking of filamentous M13 phages displaying a cell penetrating transbody and TAT peptide. PLOS ONE, 7, 1-14.

Knittelfelder R, Riemer AB, Jensen-Jarolim E, (2009). Mimotope vaccination - from allergy to cancer. Expert Opin Biol Ther, 9, 493-506.

Koivunen E, Arap W, Rajotte D, Lahdenranta J, Pasqualini R (1999). Identification of receptor ligands with phage display peptide libraries. J Nucl Med, 40, 883-8.

Lankes HA, Zanghi CN, Santos K, et al (2007). In vivo gene delivery and expression by bacteriophage lambda vectors. J Appl Microbiol, 102, 1337-49.

Li W, Joshi MD, Singhania S, Ramsey KH, Murthy AK (2014). Peptide vaccine: progress and challenges. Vaccines, $\mathbf{2}$, 515-36.

Loessner MJ, Rudolf M, Scherer S (1997). Evaluation of luciferase reporter bacteriophage $\mathrm{A} 511:: 1 \mathrm{lux} \mathrm{AB}$ for detection of Listeria monocytogenes in contaminated foods. Appl Environ Microbiol, 63, 2961-5.

Luzzago A, Felici F, Tramontano A, Pessi A, Cortese R (1993). Mimicking of discontinuous epitopes by phage-displayed peptides, I. Epitope mapping of human $\mathrm{H}$ ferritin using a phage library of constrained peptides. Gene, 128, 51-57.

Malik P, Perham RN (1997). Simultaneous display of different 
Phage Particles as Vaccine Delivery Vehicles: Concepts, Applications and Prospects

peptides on the surface of filamentous bacteriophage. Nucleic Acids Res, 25, 915-6.

Manoutcharian K, Díaz-Orea A, Gevorkian G, et al (2004). Recombinant bacteriophage-based multiepitope vaccine against Taenia solium pig cysticercosis. Vet Immunol Immunopathol, 99, 11-24.

Manoutcharian K, Terrazas LI, Gevorkian G, et al (1999). Phagedisplayed T-cell epitope grafted into immunoglobulin heavychain complementarity-determining regions: an effective vaccine design tested in murine cysticercosis. Infect Immun, 67, 4764-4770.

March JB, Clark JR, Jepson CD (2004). Genetic immunization against hepatitis $\mathrm{B}$ using whole bacteriophage $\lambda$ particles. Vaccine, 22, 1666-71.

Meij P, Leen A, Rickinson AB, et al (2002a). Identification and prevalence of $\mathrm{CD} 8+\mathrm{T}$-cell responses directed against Epstein-Bar virus-encoded latent membrane protein 1 and latent membrane protein 2. Int J Cancer, 99, 93-99.

Meij P, Vervoort MBHJ, Bloemena E, et al (2002b). Antibody responses to Epstein-Barr virus-encoded latent membrane protein-1 (LMP1) and expression of LMP1 in juvenile Hodgkin's disease. J Med Virol, 68, 370-377.

Menéndez T, Santiago-Vispo NF, Cruz-Leal Y, et al (2011). Identification and characterization of phage-displayed peptide mimetics of Neisseria meningitidis serogroup B capsular polysaccharide. Int J Med Microbiol, 301, 16-25.

Moingeon P, de Taisne C, Almond J (2002). Delivery technologies for human vaccines. Br Med Bull, 62, 29-44.

Neufeld T, Schwartz-Mittelmann A, Biran D, Ron EZ, Rishpon J (2003). Combined phage typing and amperometric detection of released enzymatic activity for the specific identification and quantification of bacteria. Anal Chem, 75, 580-5.

Odermatt A, Audige A, Frick C, et al (2001). Identification of receptor ligands by screening phage display peptide libraries ex vivo on microdissected kidney tubules. J Am Soc Nephrol, 12, 308-16.

Ou C, Tian D, Ling Y, et al (2013). Evaluation of an ompA-based phage-mediated DNA vaccine against Chlamydia abortus in piglets. Int immunopharmacol, 16, 505-10.

Pande J, Szewczyk MM, Grover, AK (2010). Phage display: Concept, innovations, applications and future. Biotechnol $A d v, \mathbf{2 8}, 849-58$.

Petrovsky N, Aguilar JC (2004). Vaccine adjuvants: Current state and future trends. Immunol cell Biol, 82, 488-96.

Purcell AW, McCluskey J, Rossjohn J (2007). More than one reason to rethink the use of peptides in vaccine design. Nat Rev Drug Disc, 6, 404-414.

Ren SX, Ren ZJ, Zhao MY, et al (2009). Antitumor activity of endogenous mFlt4 displayed on a T4 phage nanoparticle surface. Acta Pharmacol Sin, 30, 637-45.

Sartorius R, Pisu P, Apice LD, et al (2008). The use of filamentous bacteriophage fd to deliver MAGE-A10 or MAGE-A3 HLAA2-restricted peptides and to induce strong antitumor CTL responses. J Immunol, 180, 3719-28.

Saxena MV, M, Van TTH, Baird FJ, Coloe PJ, Smooker PM (2013). Pre-existing immunity against vaccine vectors friend or foe? Microbiol, 159, 1-11.

Schmelcher M, Loessner MJ (2014). Application of bacteriophages for detection of foodborne pathogens. Bacteriophage, 4.

Smith G P (1985). Filamentous fusion phage: novel expression vectors that display cloned antigens on the virion surface. Science, 228, 1315-7.

Souza APD, Haut L, Reyes-Sandoval A, Pinto, AR (2005). Recombinant viruses as vaccines against viral diseases. Brazi J Med Biol Res, 38, 509-22.

Ulivieri C, Citro A, Ivaldi F, et al (2008). Antigenic properties of HCMV peptides displayed by filamentous bacteriophages vs. synthetic peptides. Immunol Lett, 119, 62-70.

van Houten NE, Henry KA, Smith GP, Scott JK (2010). Engineering filamentous phage carriers to improve focusing of antibody responses against peptides. Vaccine, 28, 21742185.

van Houten NE, Zwick MB, Menendez A, Scott JK (2006). Filamentous phage as an immunogenic carrier to elicit focused antibody responses against a synthetic peptide. Vaccine, 24, 4188-200.

Wan Y, Yuzhang Y, Bian J, et al (2001). Induction of hepatitis B virus-specific cytotoxic $\mathrm{T}$ lymphocytes response in vivo by filamentous phage display vaccine. Vaccine, 19, 2918-23.

Wan Y, Wu Y, Zhou J, et al (2005). Cross-presentation of phage particle antigen in MHC class II and endoplasmic reticulum marker-positive compartments. Eur J Immunol, 35, 2041-50.

Wang G, Sun M, Fang J, et al (2006). Protective immune responses against systemic candidiasis mediated by phagedisplayed specific epitope of Candida albicans heat shock protein 90 in C57BL/6J mice. Vaccine, 24, 6065-6073.

Wang LF, Yu M (2004). Epitope identification and discovery using phage display libraries: applications in vaccine development and diagnostics. Curr Drug Targets, 5, 1-15.

Wang Y, Su Q, Dong S, et al (2014). Hybrid phage displaying SLAQVKYTSASSI induces protection against Candida albicans challenge in BALB/c mice. Hum Vaccin Immunother, 10, 1057-63.

Willis AE, Perham RN, Wraith D (1993). Immunological properties of foreign peptides in multiple display on a filamentous bacteriophage. Gene, 128, 79-83.

Winter G, Griffiths AD, Hawkins RE, Hoogenboom HR (1994). Making antibodies by phage display technology. Anпи Rev Immunol, 12, 433-55.

Wright A, Hawkins CH, A“ngga ${ }^{\circ}$ rd EE, Harper DR (2009). A controlled clinical trial of a therapeutic bacteriophage preparation in chronic otitis due to antibiotic-resistant Pseudomonas aeruginosa; a preliminary report of efficacy. Clin Otolaryngol, 34, 349-57.

Wu Y, Wan Y, Bian J, et al (2002). Phage display particles expressing tumor-specific antigens induce preventive and therapeutic anti-tumor immunity in murine P815 model. Int J Cancer, 98, 748-53.

Yang J, Li Y, Jin S, et al (2015). Engineered biomaterials for development of nucleic acid vaccines. Biomater Res, 19.

Yokoyama-Kobayashi M, Kato S (1994). Recombinant f1 phage-mediated transfection of mammalian cells using lipopolyamine technique. Anal Biochem, 223, 130-134. 